\title{
Toxicity of Cadmium to Saltwater Plankton and the Relative Significance of Aquatic Exposure
}

Zaosheng Wang · Changzhou Yan •

Xian Zhang · Yijun Yan

Received: 1 August 2008/Accepted: 15 October 2008

(C) Springer Science+Business Media, LLC 2009

This article has been retracted.

This article has been retracted.

Z. Wang $(\bowtie) \cdot$ C. Yan $(\bowtie) \cdot X$. Zhang $\cdot$ Y. Yan

Key Laboratory of Urban Environment and Health, Institute of

Urban Environment, Chinese Academy of Sciences, Xiamen

361003, China

e-mail: zswang@iue.ac.cn

C. Yan

e-mail: czyan@iue.ac.cn 